\title{
No Significant Difference Between Intramedullary and Extramedullary Button Fixation for Distal Biceps Brachii Tendon Rupture After Cyclic Loading in a Cadaver Model
}

\author{
Aditi Majumdar, M.D., Christina Salas, Ph.D., William Chavez, B.S., \\ Christopher Bankhead, M.D., Tony J. Sapradit, B.S., Deana Mercer, M.D., \\ Daniel C. Wascher, M.D., and Dustin L. Richter, M.D.
}

\begin{abstract}
Purpose: To biomechanically compare intramedullary (IM) versus extramedullary (EM) distal biceps button fixation under cyclic loading conditions, which is most representative of postoperative physiologic status. Methods: This controlled laboratory study used 13 fresh-frozen matched paired cadaver elbows. One specimen from each pair was randomized to either IM (unicortical) or EM (bicortical) distal biceps button fixation via onlay technique. A servohydraulic actuator was used to cycle each specimen from full extension to $90^{\circ}$ of flexion at $0.5 \mathrm{~Hz}$ for 3,000 cycles. All specimens were subsequently loaded to failure to simulate an acute postoperative load. Results: During cyclic loading, the mean change in force from cycle 5 to cycle 3000 was $2.1 \pm 3.2 \mathrm{~N}$ for the IM group and $0.6 \pm 4.2 \mathrm{~N}$ for the EM group $(P=.19)$. The increase in tendon gap for the IM group was $1.02 \mathrm{~mm}$ and for the EM group was $1.83 \mathrm{~mm}(P=.37)$. During failure loading, the IM group had a mean failure load of $154.9 \pm 44.5 \mathrm{~N}$ and the EM group a mean failure load of $191.1 \pm 62.6 \mathrm{~N}$ $(P=.16)$. Conclusions: No significant differences exist between the IM and EM techniques in loss of force and tendon gap formation under cyclic loading or load to failure conditions. Thus, IM fixation may adequately facilitate optimal bonetendon apposition, with less risk of iatrogenic injury to the posterior interosseous nerve that can be seen with bicortical extramedullary fixation. Clinical Relevance: The most common major complication following distal biceps repair is PIN palsy. IM fixation may be sufficient in facilitating optimal bone-tendon apposition and healing with onlay technique, while minimizing risk of iatrogenic PIN injury associated with EM fixation.
\end{abstract}

A cute distal biceps brachii tendon ruptures are increasingly common injuries affecting active, young male patients. ${ }^{1-3}$ In recent years, improved clinical and diagnostic modalities in combination with a more active and working population have led to an increased distal biceps rupture estimated incidence of 2.55 per 100,000 patients per year. Distal biceps tendon ruptures most frequently occur as an avulsion injury to the tendinous insertion at the bicipital tuberosity. ${ }^{4}$

From the Department of Orthopaedics $\theta$ Rehabilitation (A.M., C.S., C.B. D.M., D.C.W., D.L.R.), School of Medicine (W.C.), Department of Mechanical Engineering (C.S., T.J.S.), and Center for Biomedical Engineering (C.S.), The University of New Mexico, Albuquerque, New Mexico, U.S.A.

The authors report the following potential conflicts of interest or sources of funding: D.L.R. reports nonfinancial support from Arthrex, during the conduct of the study, and other from Arthrex and Smith $\theta$ Nephew, outside the submitted work. D.C.W. reports grants from Arthrex and Smith $\theta$ Nephew, outside the submitted work. Full ICMJE author disclosure forms are available for this article online, as supplementary material.

Received May 4, 2020; accepted January 27, 2021.
Compared with nonoperative treatment, anatomic surgical repair of an acute distal biceps tendon rupture has been shown to reliably restore strength and endurance in elbow flexion and forearm supination as well as demonstrating greater patient subjective and functional outcome measures. ${ }^{5-8}$ Complications associated with primary anatomic repair include heterotopic ossification, radioulnar synostosis or loss of forearm rotation, lateral antebrachial cutaneous nerve or

Address correspondence to Christina Salas, Ph.D., Department of Orthopaedics $\theta$ Rehabilitation, The University of New Mexico School of Medicine, MSC10 5600, 1 University of New Mexico, Albuquerque, NM 87131. E-mail: chrsalas@salud.unm.edu

(C) 2021 THE AUTHORS. Published by Elsevier Inc. on behalf of the Arthroscopy Association of North America. This is an open access article under the CC BY-NC-ND license (http://creativecommons.org/licenses/by-nc-nd/4.0/). 2666-061X/20763

https://doi.org/10.1016/j.asmr.2021.01.022 
posterior interosseous nerve (PIN) palsy, and bicipital tuberosity fracture. ${ }^{4,9-12}$ In a recent systematic review evaluating complications following distal biceps tendon repair, the authors noted that the most common major complication was PIN palsy $(1.6 \%)$, followed by rerupture $(1.4 \%)$ and symptomatic heterotopic ossification $(0.3 \%) .{ }^{13}$ Bain et al. ${ }^{14}$ noted that in cadaveric studies the average distance from the biceps tendon to the PIN was $18 \mathrm{~mm}$, and the average distance from the PIN to a Steinman pin advanced through the proximal radius was $14 \mathrm{~mm}$.

Repair of the distal biceps tendon is well described and achievable through a variety of methods, including suture anchor fixation, interference screw fixation, the transosseous technique, and button fixation. ${ }^{2,7,15-18}$ Multiple biomechanical studies have shown distal biceps button fixation to have the greatest load to failure and stiffness when compared with bone tunnels, intraosseous screws, and suture anchors. ${ }^{6,7,19}$ Unicortical button fixation has the advantage of avoiding inadvertent injury to the PIN but may have weaker fixation properties. Previous studies have demonstrated no significant differences in mean load applied before failure between intramedullary (IM or unicortical) and extramedullary (EM or bicortical) button fixation. Histologic studies in animals have demonstrated similar tendon-bone interdigitation and fibrocartilaginous zone formation at the outer cortical surface with tendon fixation within a bone tunnel (inlay) or upon a cortical surface (onlay). ${ }^{20}$

The purpose of the current study was to biomechanically compare IM versus EM distal biceps button fixation under cyclic loading conditions, which is most representative of postoperative physiologic status. The authors hypothesized there would be no significant difference in loss of force, tendon gap formation, or ultimate failure load following cyclic loading between IM and EM button fixation with tendon onlay technique for the repair of distal biceps tendon rupture in a cadaveric model.

\section{Methods}

\section{Specimen Preparation}

Thirteen matched pairs (26 total) of fresh-frozen, cadaveric elbows extending from mid-humerus to mid-forearm were used in this study. Specimens were purchased from Science Care (Phoenix, AZ) and United Tissue Network (Norman, OK). Exclusion criteria included specimens that had previous trauma or history of previous surgery affecting the upper extremity. Specimens were further excluded if, upon dissection, there was indication of injury to the biceps tendon or injury to the stabilizing ligaments of the elbow. We controlled for tissue variability by critically examining cause of death and excluding donors who had received radiation or chemotherapy as treatment for cancer as this has been shown to reduce the mechanical competency of the soft tissue, which was the subject of the current analysis. The final specimens included 13 matched pairs ( 8 female, 5 male) with a mean age of 60.2 years.

One specimen from each pair was randomized into 1 of 2 groups: IM (unicortical) or EM (bicortical) button reconstructions. Before anatomic dissection and testing, specimens were thawed for 24 hours at room temperature. Skin, fat, and muscle were removed from each specimen, leaving the biceps tendon, interosseous ligament, elbow ligaments, and capsule intact. The proximal humerus and distal ulna from each specimen was potted with a urethane casting resin (Smooth-Cast, Smooth-On Inc., Macungie, PA) for mounting to the test fixture.

The distal biceps repair with button was performed as described by Bain et al. ${ }^{14}$ The biceps tendon insertion on the bicipital tuberosity was identified and sharply transected with a knife, releasing it from its distal attachment. Each distal biceps tendon was secured with a single nonabsorbable polyester suture, No. 2 FiberLoop (Arthrex, Naples, FL) using a standard whipstitch. The whipstitch was started approximately $3 \mathrm{~cm}$ from the distal tendon edge. A total of 6 locking throws were advanced toward the distal end of the tendon, spanning the distal $3 \mathrm{~cm}$ of tendon. The suture limbs were then threaded through a 2.6- $\times 12-\mathrm{mm}$, titanium BicepsButton (Arthrex) in the usual fashion. All specimen preparation and fixation was performed by a sports medicine fellowship-trained orthopaedic surgeon (D.L.R.) and 2 senior residents (A.M., C.B.).

\section{Intramedullary (Unicortical)}

For the IM technique, the bicipital tuberosity was exposed with the forearm in maximum supination. A 3.2-mm drill bit was then drilled through volar cortex only into the central aspect of the bicipital tuberosity with a slightly ulnar trajectory. The button was then inserted through the drill hole into the intramedullary canal. The button was deployed and the tendon was advanced down to the cortical surface and manually tensioned for an onlay technique. Deployment and tensioning occurred in maximal supination and approximately $45^{\circ}$ of elbow flexion. One suture limb was then passed through the tendon with a free needle and tied.

\section{Extramedullary (Bicortical)}

For the EM technique, the bicipital tuberosity was prepared by placing the forearm in maximum supination. A 3.2-mm drill bit was then drilled through both cortices at the central aspect of the bicipital tuberosity, with the trajectory of the pin slightly ulnar. The button was deployed through the hole and positioned against the posterior radial cortex, in an extramedullary, 
bicortical position. The tendon was advanced down to the cortical surface and manually tensioned for an onlay technique. Deployment and tensioning occurred in maximal supination and approximately $45^{\circ}$ of elbow flexion. One suture limb was then passed through the tendon with a free needle and tied.

\section{Biomechanical Testing}

All testing was performed by a Ph.D. biomedical engineer (C.S.). A custom test fixture was designed to allow for elbow motion from $90^{\circ}$ of flexion to full extension during cyclic loading, similar to that described by Savin et al. ${ }^{2}$ For ramped failure testing, the fixture enabled locking the elbow at $90^{\circ}$ of flexion. The humerus was fixed to a vertical support plate through the humerus resin block. The ulna resin block was inserted into a 3D-printed support box and weighted to approximately $9 \mathrm{~N}$ to simulate the mass of the distal forearm and hand. The radius was permitted to move freely. The biceps tendon was fixed proximally with a locking nonabsorbable whipstitch to an overlayed nylon strap. The nylon was attached to a rigid cable, routed through a pulley, and was fixed to the servohydraulic test frame actuator for loading (Mini Bionix 858; MTS Systems, Eden Prairie, MN).

Based on previously published methodology, specimens were cyclically loaded from full extension to $90^{\circ}$ of elbow flexion at $0.5 \mathrm{~Hz}$ for 3000 cycles to simulate active motion in the immediate postoperative period (Fig 1). ${ }^{2}$ Cyclic motion was produced through displacement control of the actuator from $0 \mathrm{~mm}$ (full extension) to $60 \mathrm{~mm}\left(90^{\circ}\right.$ flexion $)$. This mode of loading was selected to simulate a constant displacement from biceps brachii muscle contracture. The maximum force from force-displacement hysteresis data were analyzed between cycle 5 and cycle 3000 to evaluate the loss of fixation. Specimens that did not fail in 3000 cycles were then ramped at $1 \mathrm{~mm} / \mathrm{s}$ with the elbow fixed at $90^{\circ}$ until failure occurred.

\section{Image Analysis for Failure Determination}

A retroreflective marker of known diameter was placed on the surface of each ulna, on the side aligning with the right side of the test frame. A surgical marking pen was used to place a mark on the surface of the repaired biceps tendon. A Nikon digital camera was positioned normal to the ulna marker to capture planar images of tendon displacement. One image was taken of the specimen before cyclic loading, with the elbow held at $90^{\circ}$ flexion, and a subsequent image was taken of the specimen post cyclic loading, with the elbow flexed in the same position. Image $\mathrm{J}$ image processing software was used to quantify relative displacement of the mark on the tendon from the superior edge of the ulna, before and after cyclic loading, as a measure of tendon gapping. ${ }^{21}$ All images were individually calibrated using the retroreflective ulnar marker.

\section{Statistical Analysis}

Outcome measures from cyclic testing included a loss of maximum force at $90^{\circ}$ flexion as calculated by the difference of the maximum force at cycle 3000 from the maximum force at cycle 5. Tendon gapping was quantified using image analysis to identify potential cause of loss of force. This was defined as a difference in displacement of the tendon marker from pre-cyclic to post-cyclic loading. Outcome measures from ramped failure testing included the ultimate force before failure, stiffness, and failure mode. Stiffness of the construct, calculated as the slope of the most linear region of the force-displacement curve, was evaluated as a measure of fixation stability. In addition, all modes of failure were evaluated, to include suture and tendon creep, displacement of bone-tendon interface, fracture, and button pullout. Means and standard deviations were calculated and reported.

Descriptive statistics for the data set were generated using SPSS, version 25 (IBM Corp., Armonk, NY). The analysis was performed by a member of our
Fig 1. A graphical representation of the cyclic testing methodology. Specimens were cycled from $90^{\circ}$ of flexion to full extension using a displacement-controlled protocol simulating fixed biceps muscle contraction at $0.5 \mathrm{~Hz}$ for 3000 cycles.

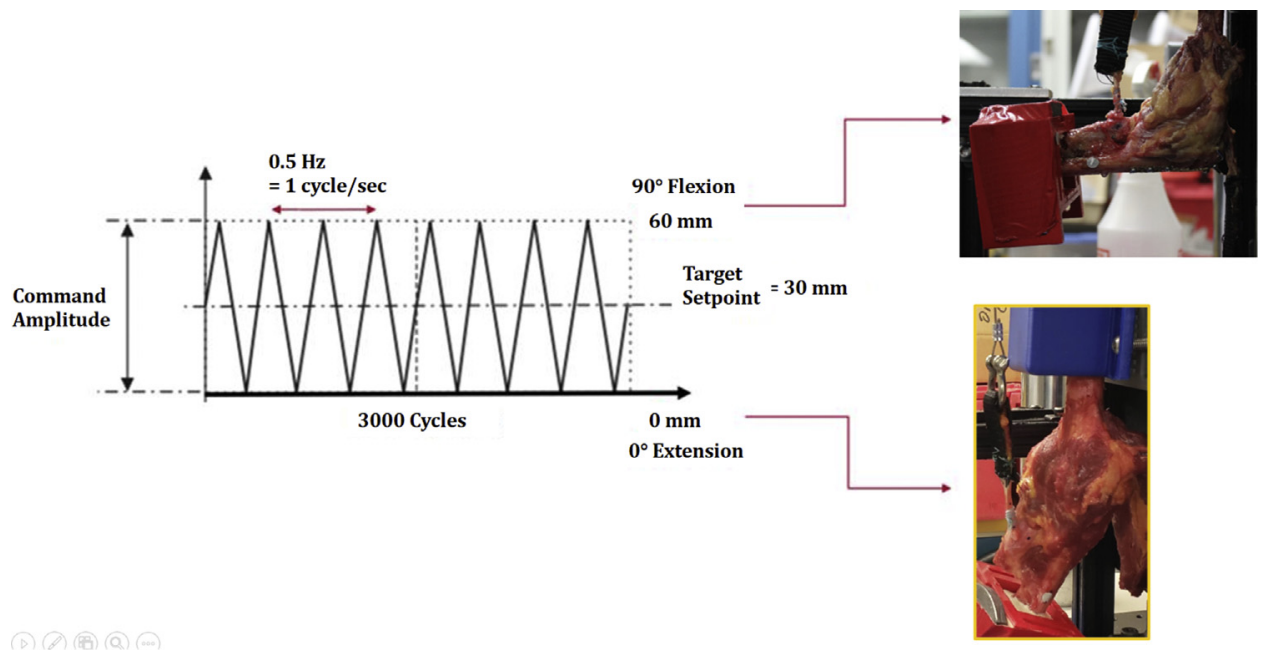


department's research division with a strong background in statistics (see the Acknowledgments section). A box plot graphical analysis was used for describing the behavior of the data (interquartile range) and to identify outliers in the data set. Any data point outside of the upper outer fence (quartile 3+3*interquartile range) or the lower outer fence (quartile $1-3 *$ interquartile range) was excluded as an outlier. This was confirmed using $\mathrm{R}$ statistical software and the Grubbs test to detect outliers. A Shapiro-Wilk test was used to determine normality of the data set. If the data were determined to be normally distributed, a paired, 2 -tailed Student $t$ test was used to determine statistical significance at an alpha of 0.05 . If the data were not normally distributed, a Mann-Whitney $U$ test was used.

\section{Results}

The box plot analysis showed that the loss in force (from cycle 5 to cycle 3000) for specimen 12 and 13, EM data, were outliers. This was also confirmed using the Grubbs test for outliers in $\mathrm{R}$ statistical software, indicating that it was safe and appropriate to eliminate these data points. These data points and the data for their matched pair (IM) were removed from final cyclic data analysis. The ultimate force and stiffness data from these specimens were included in the ramped failure test analysis. The Shapiro-Wilk test found that the data set was normally distributed, thus a paired, 2-tailed, Student $t$ test was used for analysis of statistical difference. The data collected and analyzed in this study can be found in Table 1 .

\section{Cyclic Testing}

The mean maximum force at $90^{\circ}$ flexion during cycle 3000 was $13.5 \pm 2.5 \mathrm{~N}$ for IM and $12.2 \pm 3.9 \mathrm{~N}$ for EM button fixation. The mean maximum force at $90^{\circ}$ flexion during cycle 5 was $11.4 \pm 2.1$ for IM and $11.6 \pm 2.6 \mathrm{~N}$ for EM button fixation. The loss of force during cyclic loading was $2.1 \pm 3.2 \mathrm{~N}$ for IM and $0.6 \pm 4.2 \mathrm{~N}$ for EM fixation. There was no statistical difference in loss of force between IM and EM button fixation during cyclic loading $(P=.19)$. The tendon gap increased by $1.0 \mathrm{~mm}$ for the IM fixation group and 1.8 $\mathrm{mm}$ for the EM fixation group $(P=.37$ between groups).

\section{Failure Testing}

The mean ultimate force before failure was $154.9 \pm 44.5 \mathrm{~N}$ for $\mathrm{IM}$ and $191.1 \pm 62.6 \mathrm{~N}$ for $\mathrm{EM}$ button fixation. There was no statistical difference in ultimate failure force between IM and EM button fixation $(P=.16)$. The stiffness was $7.2 \pm 2.0 \mathrm{~N} / \mathrm{mm}$ for IM and $9.0 \pm 2.6 \mathrm{~N} / \mathrm{mm}$ for EM button fixation. There was no statistical difference in stiffness between IM and EM fixation $(P=.07)$.

\section{Modes of Failure}

During failure testing, the IM and EM specimens experienced 4 modes of failure: tendon-suture failure $(\mathrm{IM}=6, \mathrm{EM}=5)$, suture-button failure $(\mathrm{IM}=1$, $\mathrm{EM}=1)$, proximal interosseous membrane failure leading to radial head dislocation ( $\mathrm{IM}=4, \mathrm{EM}=3)$, or by tendon-strap failure ( $\mathrm{IM}=2, \mathrm{EM}=4)$ (Fig 2). No failures of either IM or EM specimens were secondary to button pullout or fracture.

\section{Discussion}

The most important finding of this study is that we observed no significant differences between intramedullary versus extramedullary cortical button fixation in tendon elongation and tendon gap formation at the bone-tendon interface under cyclic loading conditions, using a $9 \mathrm{~N}$ preload. This indicates that both

Table 1. Data Table Showing Loss of Force (N) from Cycle 5 to Cycle 3000, the Ultimate Failure Force (N), and Stiffness (N/mm) of the Loaded Specimens With Distal Biceps Button Repair Using a Unicortical or Bicortical Button Technique

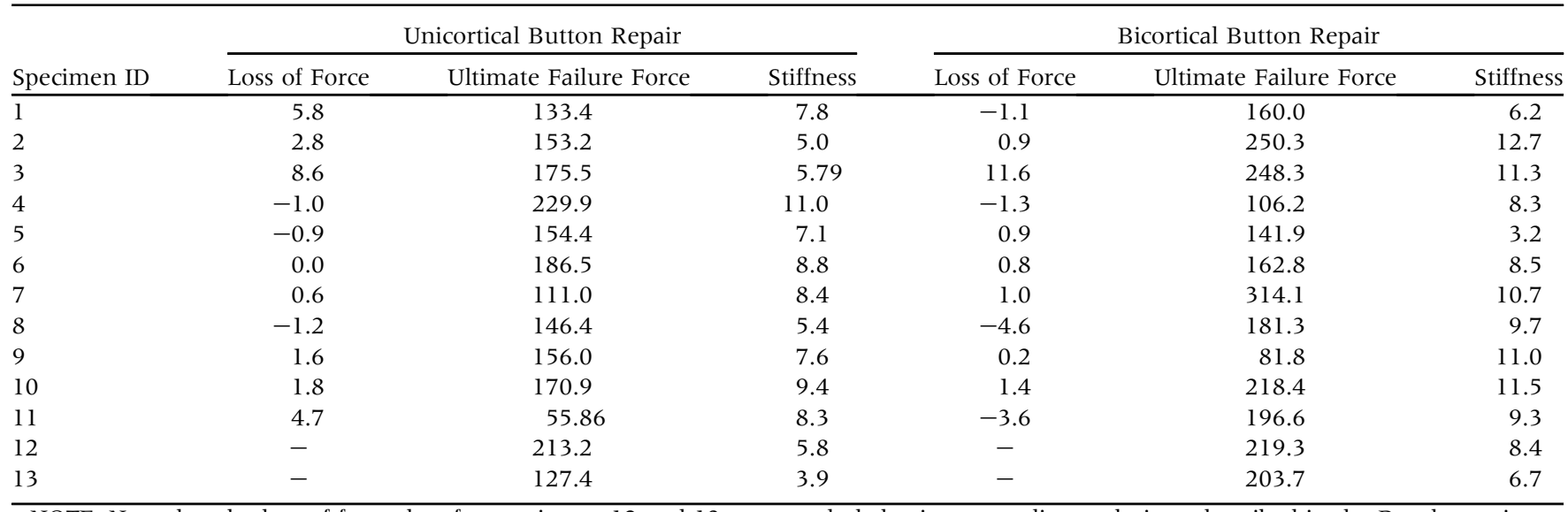



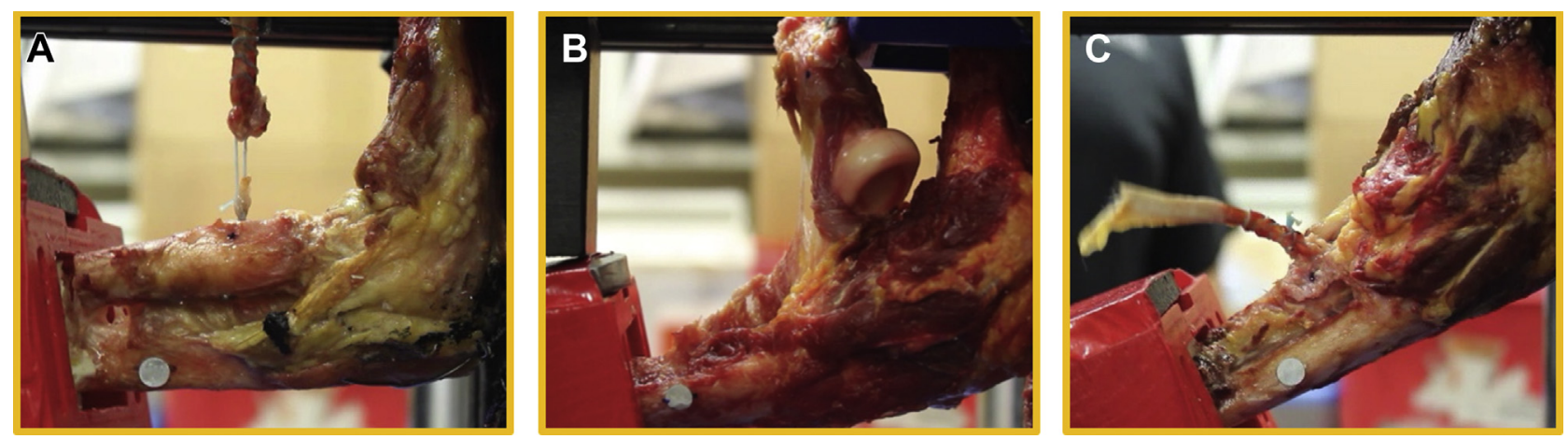

Fig 2. Modes of failure observed in the present study: (A) tendon-suture failure or suture-button failure; (B) proximal interosseous membrane failure leading to radial head dislocation; (C) tendon-strap failure.

techniques maintained an equivalent stabilization. Thus, a theoretic advantage of IM fixation may be equivalent bone-tendon apposition with lower risk of PIN capture compared with extramedullary fixation when following a single incision approach; however, further clinical studies are warranted.

Acute anatomic surgical repair of distal biceps brachii tendon has been shown to improve functional outcomes, strength, and muscle endurance after distal biceps rupture. ${ }^{1,16,22,23}$ Cortical button fixation has been shown to outperform bone tunnels, intraosseous screws, and suture anchors in biomechanical studies. ${ }^{7,12,16,19}$ As noted previously, the most common major complication following surgery is PIN palsy, and unicortical button fixation may have the advantage of avoiding inadvertent injury to the PIN. ${ }^{13}$ While these biomechanical studies were most demonstrative of time-zero construct strength and stability, there is minimal evidence evaluating techniques under cyclic loading conditions. By subjecting each specimen to cyclic load, as modeled per a previously validated protocol, we attempted to simulate normal postoperative distal biceps repair demands. ${ }^{2}$ Tendon gap formation is a reported disadvantage to cortical button fixation. In theory, a construct susceptible to gap formation may limit biologic incorporation, despite a greater load to failure on biomechanical testing. ${ }^{24}$ Despite a body of literature investigating tendon gap formation, the exact clinical significance is unknown. ${ }^{7,16,25,26}$ Previous studies have shown decreased gapping and motion to theoretically permit early, aggressive range of motion with no sacrifice in bone-tendon healing. Hahn et al. ${ }^{27}$ noted 17-mm gapping with tendon elongation following cyclic loading of porcine flexor digitorum tendons, promoting the performance of preloading to minimize this effect. By studying repaired tendon behavior under simulated physiologic cyclic load conditions, protocols to support the implementation of early postoperative elbow range of motion can be designed. Similarly, IM fixation may be sufficient in facilitating optimal bone-tendon apposition and healing, while minimizing risk of iatrogenic PIN injury that can be associated with EM fixation. ${ }^{16}$

Our study exclusively used onlay technique for both IM and EM distal biceps fixation. In an animal histologic, biomechanical, and radiographic analysis of proximal biceps tenodesis, Tan et al. ${ }^{20}$ demonstrated cortical tendon to bone healing profiles are similar for both cortical surface and bone tunnel fixation techniques. In the study, 32 rabbits underwent proximal biceps tenodesis with bone tunnel versus cortical surface technique, yielding similar tendon-bone interdigitation and early fibrocartilaginous zone formation on the outer cortical surface, and minimal intramedullary healing. Histologic studies have demonstrated tendon-to-bone healing with interference screw fixation. In this study we did not observe any statistical difference in bone-tendon displacement as a measure of loss of fixation during cyclic loading; furthermore, bone tunnel fixation was not a variable.

In a cadaveric series by Greenberg et al., ${ }^{28}$ in comparison with suture anchors and bone bridge technique, IM button fixation exhibited greatest pullout strength, yielding greater than twice the strength of either of the 2 other techniques. Similarly, Mazzoca et al. ${ }^{7}$ demonstrated unicortical button fixation to possess greatest load to failure in comparison to bone tunnels, interference screw fixation, and suture anchor techniques. However, their technique placed the button on the far cortex and used an inlay technique with placing the graft in the medullary canal. Another recent study showed that all-suture anchors were biomechanically equivalent at time zero to titanium suture anchors for onlay distal biceps tendon repair. ${ }^{17}$ The current study showed a mean load to failure of $154.9 \pm 44.5 \mathrm{~N}$ with IM button fixation and $191.1 \pm 62.6 \mathrm{~N}$ with EM button fixation. There was no significant difference $(P=.16)$ in failure load between the 2 groups, indicating an equivalent response in the event of an acute injury. Our results are congruent with maximum generated in vivo eccentric forces of the native human elbow, proposed at 100 to $200 \mathrm{~N}^{22}$ Similarly, Siebenlist et al. ${ }^{16}$ 
demonstrated no significant difference in load to failure between single intramedullary and single extramedullary cortical button fixation in a linear load to failure analysis of 12 cadaveric specimens $(P=.081)$.

In general, modes of failure in this study were similar to those of previous cadaveric models, and included suture failure (tendon-suture, suture-button, tendon-strap interfaces) and proximal interosseous membrane failure leading to radial head dislocation. ${ }^{2,16,25}$ However, in contrast to previous cadaveric studies, fracture of the bicipital tuberosity and button pullout were not observed as modes of failure. ${ }^{16}$ This difference, while multifactorial, may be attributed to differences in testing apparatus, radius restraint, bone density, and implant trajectory and placement.

\section{Limitations}

Our study has several limitations. The primary limitations to our study include factors that affect the similarity of our study specimens to in vivo function of these surgical repairs. Cadaver studies generally produce slightly different results than living tissue. These time-zero studies simulate a worst-case scenario situation providing evidence of potential implant behavior without the effects of healing. Although we would anticipate that tissue healing would only reinforce the strength of IM and EM fixation using an onlay technique, this has yet to be confirmed with in vivo studies. Some additional factors include age and sex of our cadaveric specimens. The average age of specimens used in this study (60.2 years) was older than the average for distal biceps tendon rupture (46.3 years), although not unreasonable for this condition (range, 20-72 years). ${ }^{4}$ Both advanced age and female sex may negatively affect bone mineral density, which can contribute significantly to differences in load to failure. It is important to note that while female and older age specimens were used in this study, there were no observed failures due to fracture or button pullout. Another limitation of our study is that the motion used for both cyclic loading and load to failure were primarily uniplanar. While we attempted to create a more representative clinically applicable loading orientation by not restraining the radius, this does not perfectly replicate how the arm moves in vivo, and could be a confounding factor in extrapolating our study data and making it more generalizable. Finally, another limitation is in the sample size used for our experimental test. A post-hoc power analysis was performed using mean and standard deviation data collected from our study to determine the minimum sample size needed in order to reject the null hypothesis that there would be no detectable difference between intramedullary and extramedullary fixation. This analysis showed that a minimum sample size of 72 specimens per group would be needed to detect a difference between the two groups with a power of 0.95 . Although we were unable to include 72 specimens in each group for the present study, we included a similar number to those studies to which we have compared our aforementioned outcomes. Furthermore, we suggest that such a large sample size indicates that if a difference is detected, even if real, it is not clinically relevant.

\section{Conclusions}

No significant differences exist between the IM and EM techniques in loss of force and tendon gap formation under cyclic loading or load to failure conditions. Thus, IM fixation may adequately facilitate optimal bone-tendon apposition, with less risk of iatrogenic injury to the PIN that can be seen with bicortical extramedullary fixation.

\section{Acknowledgments}

The authors thank Ann Laurie Wells, Ph.D., for her assistance with statistical analysis.

\section{References}

1. Athwal GS, Steinmann SP, Rispoli DM. The distal biceps tendon: Footprint and relevant clinical anatomy. J Hand Surg 2007;32:1225-1229.

2. Savin DD, Piponov H, Watson JN, et al. Biomechanical evaluation of distal biceps tendon repair using tension slide technique and knotless fixation technique. Int Orthop 2017;41:2565-2572.

3. Kelly MP, Perkinson SG, Ablove RH, Tueting JL. Distal biceps tendon ruptures: An epidemiological analysis using a large population database. Am J Sports Med 2015;43: 2012-2017.

4. Belli P, Costantini M, Mirk P, Leone A, Pastore G, Marano P. Sonographic diagnosis of distal biceps tendon rupture: A prospective study of 25 cases. J Ultrasound Med 2001;20:587-595.

5. Hetsroni I, Pilz-Burstein R, Nyska M, Back Z, Barchilon V, Mann G. Avulsion of the distal biceps brachii tendon in middle-aged population: is surgical repair advisable? A comparative study of 22 patients treated with either nonoperative management or early anatomical repair. Injury 2008;39:753-760.

6. Baker BE, Bierwagen D. Rupture of the distal tendon of the biceps brachii. Operative versus non-operative treatment. J Bone Joint Surg Am 1985;67:414-417.

7. Mazzocca AD, Burton KJ, Romeo AA, Santangelo S, Adams DA, Arciero RA. Biomechanical evaluation of 4 techniques of distal biceps brachii tendon repair. Am J Sports Med 2007;35:252-258.

8. Morrey BF, Askew LJ, An KN, Dobyns JH. Rupture of the distal tendon of the biceps brachii. A biomechanical study. J Bone Joint Surg Am 1985;67:418-421.

9. Cain RA, Nydick JA, Stein MI, Williams BD, Polikandriotis JA, Hess AV. Complications following distal biceps repair. J Hand Surg 2012;37:2112-2117.

10. Dunphy TR, Hudson J, Batech M, Acevedo DC, Mirzayan R. Surgical treatment of distal biceps tendon 
ruptures: An analysis of complications in 784 surgical repairs. Am J Sports Med 2017;45:3020-3029.

11. Huynh T, Leiter J, MacDonald PB, et al. Outcomes and complications after repair of complete distal biceps tendon rupture with the cortical button technique. JB JS Open Access 2019;4.

12. Shields E, Olsen JR, Williams RB, Rouse L, Maloney M, Voloshin I. Distal biceps brachii tendon repairs: A singleincision technique using a cortical button with interference screw versus a double-incision technique using suture fixation through bone tunnels. Am J Sports Med 2015;43:1072-1076.

13. Amarasooriya M, Bain GI, Roper T, Bryant K, Iqbal K, Phadnis J. Complications after distal biceps tendon repair: A systematic review. Am J Sports Med 2020;48:3103-3111.

14. Bain GI, Prem H, Heptinstall RJ, Verhellen R, Paix D. Repair of distal biceps tendon rupture: A new technique using the endobutton. J Shoulder Elbow Surg 2000;9:120-126.

15. Camp CL, Voleti PB, Corpus KT, Dines JS. Single-incision technique for repair of distal biceps tendon avulsions with intramedullary cortical button. Arthrosc Tech 2016;5: e303-307.

16. Siebenlist S, Lenich A, Buchholz A, et al. Biomechanical in vitro validation of intramedullary cortical button fixation for distal biceps tendon repair: A new technique. Am J Sports Med 2011;39:1762-1769.

17. Otto A, Mehl J, Obopilwe E, et al. Biomechanical comparison of onlay distal biceps tendon repair: All-suture anchors versus titanium suture anchors. Am J Sports Med 2019;47:2478-2483.

18. Kodde IF, Baerveldt RC, Mulder PGH, Eygendaal D, van den Bekerom MPJ. Refixation techniques and approaches for distal biceps tendon ruptures: A systematic review of clinical studies. J Shoulder Elbow Surg 2016;25:e29-e37.

19. Kettler M, Lunger J, Kuhn V, Mutschler W, Tingart MJ. Failure strengths in distal biceps tendon repair. Am J Sports Med 2007;35:1544-1548.
20. Tan H, Wang D, Lebaschi A, et al. Comparison of bone tunnel and cortical surface tendon-to-bone healing in a rabbit model of biceps tenodesis. J Bone Joint Surg 2018;100:479-486.

21. Eyes in the Sky II. Week 2: Analyzing Change Over Time. "Getting to Know Measuring in ImageJ." TERC, Inc., Cambridge, MA, 2020, serc.carleton.edu/eyesinthesky2/ week2/get_to_know_imagej.html. Accessed December 9, 2020.

22. Klonz A, Loitz D, Wöhler P, Reilmann H. Rupture of the distal biceps brachii tendon: Isokinetic power analysis and complications after anatomic reinsertion compared with fixation to the brachialis muscle. J Shoulder Elbow Surg 2003;12:607-611.

23. Schmidt CC, Brown BT, Qvick LM, Stacowicz RZ, Latona CR, Miller MC. Factors that determine supination strength following distal biceps repair. J Bone Joint Surg Am 2016;98:1153-1160.

24. Idler CS, Montgomery WH, Lindsey DP, Badua PA, Wynne GF, Yerby SA. Distal biceps tendon repair: A biomechanical comparison of intact tendon and 2 repair techniques. Am J Sports Med 2006;34:968-974.

25. Siebenlist S, Buchholz A, Zapf J, et al. Double intramedullary cortical button versus suture anchors for distal biceps tendon repair: A biomechanical comparison. Knee Surg Sports Traumatol Arthrosc 2015;23:926-933.

26. Spang JT, Weinhold PS, Karas SG. A biomechanical comparison of EndoButton versus suture anchor repair of distal biceps tendon injuries. I Shoulder Elbow Surg 2006;15:509-514.

27. Hahn JM, Inceoğlu S, Wongworawat MD. Biomechanical comparison of Krackow locking stitch versus nonlocking loop stitch with varying number of throws. Am J Sports Med 2014;42:3003-3008.

28. Greenberg JA, Fernandez JJ, Wang T, Turner C, EndoButton-assisted repair of distal biceps tendon ruptures. J Shoulder Elbow Surg 2003;12:484-490. 